\title{
Eye Segment
}

National Cancer Institute

\section{Source}

National Cancer Institute. Eye Segment. NCI Thesaurus. Code C33525.

One of two portions (anterior or posterior) of the eye. 